\title{
Actividad repelente e insecticida de hojas, flores y extractos de llama del bosque (Spathodea campanulata B.), en gorgojos de granos almacenados (Sitophilus zeamais M.)
}

\section{Repellent and insecticide activity of leaves, flowers and extracts of African tulip tree (Spathodea campanulata B.), in stored grain weevils (Sitophilus zeamais M.)}

\author{
Reynaldo Alarcón ${ }^{1}$; Thomas Guzmán²; José Penieres ${ }^{3}$; Rosa Navarrete ${ }^{4}$. \\ ${ }^{1}$ Centro Universitario del Sur Occidente (CUNSUROC), \\ Universidad de San Carlos de Guatemala.reymazate@gmail.com \\ ${ }^{2}$ Instituto Tecnológico de Costa Rica. \\ ${ }^{3}$ Facultad de Estudios Superiores, Cuautitlán, Campo 1, UNAM. \\ ${ }^{4}$ Unidad de Granos y Semillas (UNIGRAS) UNAM, México.
}

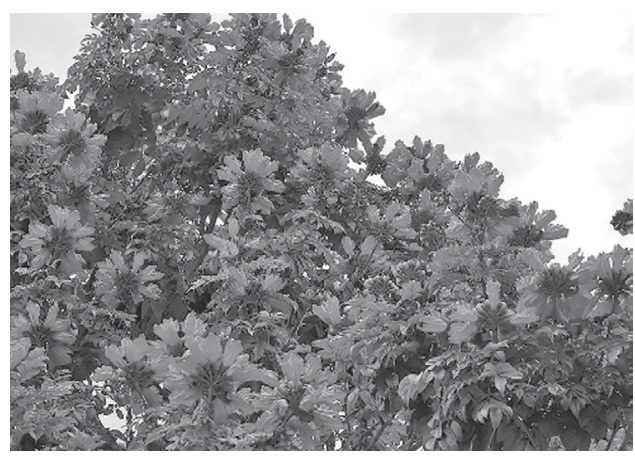

\begin{abstract}
RESUMEN
El gorgojo del maíz (Sitophilus zeamais Motschulsky. Coleóptera: Curculionidae), es una de las principales plagas del grano en "post cosecha". Las pérdidas anuales de post cosecha de cultivos de cereales son el resultado de daños de insectos, degradación microbiana y otros factores, se estima de 10 al $25 \%$ de pérdida de producción a nivel mundial. Tradicionalmente estas plagas se controlan con insecticidas químicos que si no se manejan adecuadamente, ocasionan problemas de contaminación ambiental y residuos en alimentos. Como una alternativa está el uso de insecticidas naturales que son menos tóxicos y pueden ser elaborados por los agricultores. Entre los insecticidas naturales están los compuestos derivados de nicotina, piretrinas naturales, neem (Azaradichtina) y las hojas, flores y extractos del árbol llama del bosque, que pueden constituir una alternativa para el control de plagas en granos almacenados como el gorgojo del maíz. En esta investigación se realizaron ensayos para determinar la actividad repelente e insecticida de hojas y flores de llama del bosque, así como de los extractos respectivos, en una cámara con túnel de viento del laboratorio de ecología química de insectos de ECOSUR, Tapachula, Chiapas, México. Con base en los resultados obtenidos se determinó efecto repelente de flores y hojas sobre gorgojos del maíz en concentración de 2 g/200 g de maíz a las 2 y 17 horas de aplicación. La mayor actividad insecticida se presentó a las 12 horas de la aplicación de extractos y el mejor efecto insecticida fue con el extracto de flores en concentraciones de 2.0 y $1.5 \mathrm{mg} / \mathrm{ml}$.
\end{abstract}

Palabras clave: gorgojo del maíz, llama del bosque, repelente, insecticida, extractos.

Recibido: 2 de marzo del 2016

Aceptado: 28 de septiembre del 2016
ABSTRACT
The maize weevil (Sitophilus zeamais Motsch. Coleóptera: Curculionidae), is a one of the main pest of the corn during postharvest. The annual loss of cereal crops during postharvest are results of insect damage, microbial degradation and others factors, the estimate between 10 to $25 \%$ of yield global loss. Traditionally the control of these pests is with chemical insecticides, that is not appropriate management, produces environmental problems and food residual insecticide. One alternative is to use natural insecticides that are friendlier to the environment and are more feasible to make in the field. Between the naturals products are substances from: nicotine, natural pyrethrum, neem (azaradichtina) and the African tulip leaves and flowers (S. campanulata B.), in the same way the respective extracts. These components of the tree could be an alternative to postharvest cereals pest like the maize weevil (S. zeamais). In this research we conducted assays to determinate insecticide and repellent activity of African tulip leaves, flowers and extracts respectively in a camera with wind channel in the Chemistry-Ecology Laboratory of ECOSUR, Tapachula Chiapas, Mexico. The results obtained showed statistically the insecticide and repellent effect of flowers and leaves on the maize weevil to $2 \mathrm{~g} / 200 \mathrm{~g}$ of corn and the main activity were to 2 and 17 hours after of the application. The main insecticide extract effects were with flowers extract to 2.0 and $1.5 \mathrm{mg} / \mathrm{ml}$ solution after 12 hours of the application.

Key words: The maize weevil, postharvest, "African tulip", insecticide, repellent, extract. 
$\mathrm{E}$ ntre el 30 y 40 \% de la producción de maíz en América Latina, se pierde durante el almacenamiento (Lagunes, 1994). Una de las causas son las plagas de los granos almacenados, entre ellos, el gorgojo del maíz Sitophilus zeamais y el gorgojo de las galletas, del pan o de las drogas Stegobium paniceum, los cuales atacan a granos agrícolas en el campo y durante el almacenamiento (Lagunes, Domínguez y Rodríguez 1985; Morgan, Sherington, Gudrups, \& Bowdenm 1998).

Estos gorgojos atacan a granos de maíz, arroz, sorgo y otros cereales secos o productos de cereales en almacenamiento, tiene distribución cosmopolita en climas cálidos y un ciclo de vida de 32 a 35 días a $30^{\circ} \mathrm{C}$. Las larvas se alimentan dentro del grano reduciéndolo a polvo, pero dejando la testa; la infestación puede empezar en el campo (Saunders, Coto y King 1998). Se recomienda aplicar productos fosforados como el fosfuro de aluminio o insecticidas formulados en polvo del grupo de los organofosforados (Saunders, Coto y King 1998). Para el control de gorgojo del maíz sin insecticidas se recomienda exponer los granos infestados al sol a temperaturas de 40 a $44^{\circ} \mathrm{C}$, aunque este método no mate las larvas y huevos si hace que los adultos abandonen los granos y mezclar las semillas con arena o ceniza al 5 o $10 \%$ de concentración en peso (Tejeda, 1995). En el control biológico se recomienda el uso de parasitoides de la familia Pteromalidae-Hymenoptera (Saunders, Coto y King 1998).

La forma tradicional de controlar los insectos es mediante el uso de insecticidas químicos, los cuales se han aplicado unilateralmente en forma excesiva y repetitiva sin un manejo adecuado generando problemas de resistencia de las plagas de insectos a los insecticidas (Subramanyam \& Hagstrum, 1995); también han ocasionado el surgimiento de nuevas plagas, problemas de contaminación ambiental e intoxicaciones agudas, crónicas y detección de residuos en alimentos (García, 1997). Además, con cierta frecuencia el uso de insecticidas convencionales en áreas rurales implica un riesgo elevado debido al desconocimiento sobre su uso adecuado (Silva, Pizarro, Casals, y Berti 2003).

En el contexto del manejo integrado de plagas, los plaguicidas deben ser el último recurso empleado, debido a sus repercusiones en otros organismos y en la salud humana. Por lo tanto es necesario investigar y desarrollar sistemas alternativos de control de plagas, como es el uso de insecticidas botánicos, los cuales son más amigables con el medio ambiente y pueden ser elaborados por los mismos agricultores (Hellpap, 2000). Algunos productos naturales como cenizas, estiércoles, especies, residuos de procesamiento de aceite de oliva y neem (Azaradichta indica) ha sido utilizados para controlar las plagas de insectos de granos almacenados (Levinson \& Levinson, 1998); estos productos pueden ser tóxicos, repelentes o atrayentes. La mayoría de estos productos deben ser utilizados en altas dosis o de lo contrario no realizan un buen control.
Entre otros productos naturales utilizados para el control de plagas están las hojas y flores de llama del bosque Spathodea campanulata Beauv (Figura 1), las cuales han reportado una variedad de compuestos con propiedades fagodisuasivas, insecticidas; antiparasitarias, antimalariales y anti VIH (Makinde, Amusan \& Adesogan 1998; Davis, Pylatuik, Poradis \& Low 1998). Entre los compuestos presentes están los terpenos, ácidos orgánicos, esteroles y otros compuestos volátiles. Hay estudios que evidencian la mortalidad de un 97 "por ciento" de insectos cuando visitan las flores en busca de néctar y polen; también se registra hasta un $90 \%$ de mortalidad de larvas de insectos como Spodoptera frugiperda y la reducción de la longevidad de abejas meliponas hasta un 53\% (Portugal-Araujo 1963; Segantini, Romano, \& Ribeiro 2002; Trigo y Santos 2000). Por ello en este trabajo se evaluó la actividad insecticida bajo condiciones controladas de hojas y flores, en función de la mortalidad del gorgojo del maíz para que pueda constituirse en una alternativa para el control de dicha plaga, la cual causa serios daños al maíz almacenado (Tejada, 1995).

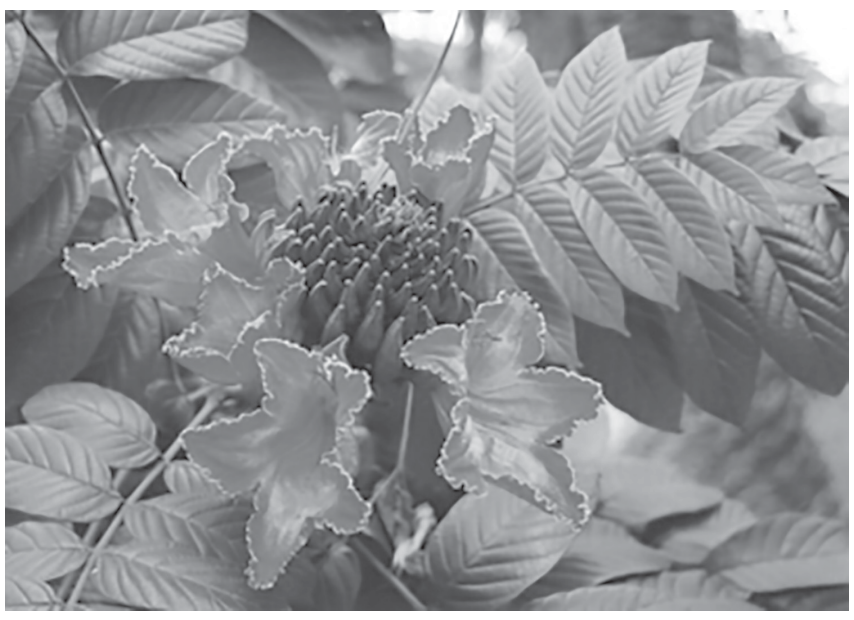

Figura 1. Inflorescencia de llama del bosque (Spathodea campanulata B.). Fotografía de Alarcón (2012).

\section{MATERIALES Y MÉTODOS}

Colecta de llama de bosque. En el ejido de Toluca, municipio de Tapachula, Chiapas, fueron colectadas hojas y flores del árbol de llama del bosque, utilizado como sombra de café y como especie ornamental. Las hojas y flores fueron cortadas en partes de aproximadamente $0.5 \mathrm{~cm}^{2}$ con una tijera, para evitar el contacto directo con el material se utilizaron guantes de caucho.

Actividad repelente de llama del bosque. Se perforaron vasos plásticos transparentes de $11 \mathrm{~cm}$ de altura y $9 \mathrm{~cm}$ de diámetro, con 16 líneas de 10 hoyos de $3 \mathrm{~mm}$ de diámetro, cada fila separada un $1 \mathrm{~cm}$ alrededor del vaso y cada hoyo separado a $0.5 \mathrm{~cm}$, el fondo se cubrió con malla plástica transparente de $2 \mathrm{~mm}$ de enrejado, con el objeto de colocar granos 
de maíz y favorecer la salida de los gorgojos por los hoyos perforados o la malla del fondo. El vaso perforado se colocó sobre otro vaso de $7 \mathrm{~cm}$ de altura y $7.5 \mathrm{~cm}$ de diámetro. Este segundo vaso se colocó en una tapa de una placa Petri de vidrio (figura 2).

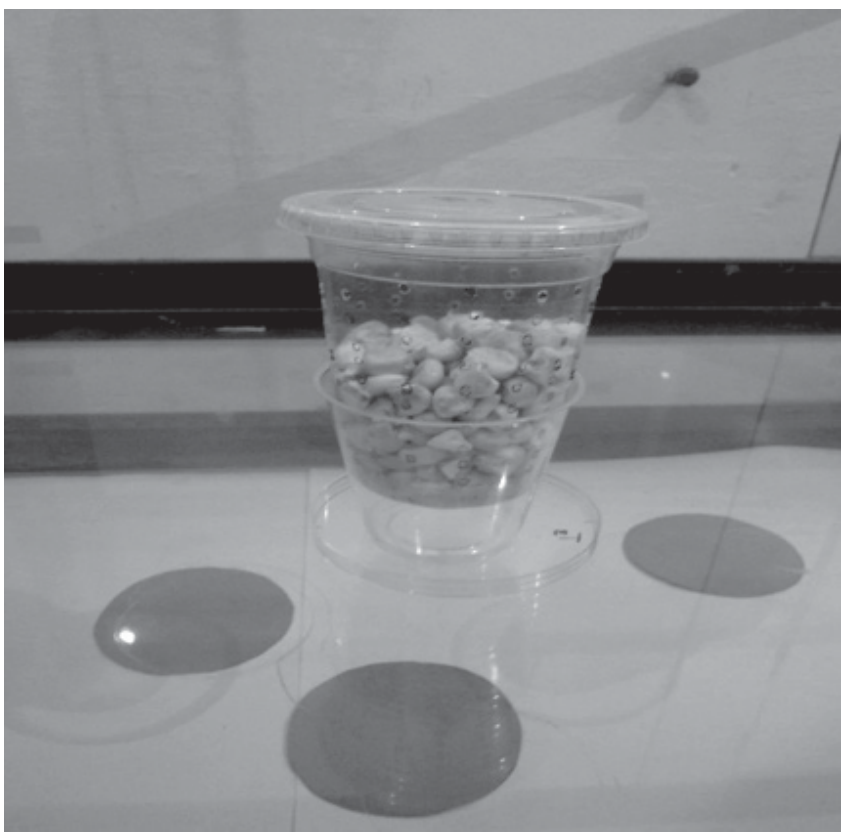

Figura 2. Equipo utilizado para ensayo de repelencia contra $S$. zeamais. Fotografía de Alarcón (2012).

Diseño experimental. Se utilizó un diseño experimental al azar con 3 tratamientos y 8 repeticiones, se colocaron 20 gorgojos adultos de S. zeamais por cada unidad experimental en $100 \mathrm{~g}$ de granos de maíz pozolero (Zea mays L.). El ensayo se realizó en el laboratorio de Ecología Química de insectos tropicales en el Colegio de la Frontera Sur (ECOSUR), a una temperatura de $27 \pm 1^{\circ} \mathrm{C}$ y $67 \pm 10 \%$ de humedad relativa.

Procedimiento. Se esterilizó el maíz pozolero durante 40 minutos a $121^{\circ} \mathrm{C}$ en autoclave. El maíz esterilizado se pesó y se colocaron $100 \mathrm{~g}$ de granos con $1 \mathrm{~g}$ de hojas o de flores picadas de llama de bosque. Posteriormente se mezclaron para cada tratamiento hojas o flores con los 100 g maíz en cada vaso perforado de cada unidad experimental. Al testigo solo se aplicó el maíz y los gorgojos.

Los gorgojos se infestaron a cada vaso mediante un embudo de vidrio de $20 \mathrm{~cm}$ de largo, con el objeto de que los gorgojos se liberaran en la parte central de los granos contenidos en el vaso perforado, aplicando la técnica de Mohan (2002). Después de liberar los gorgojos se tapó el vaso y se inició la colecta de los gorgojos que salían del vaso perforado.

Los gorgojos que salieron de los vasos perforados y los que se atraparon en el vaso inferior fueron contados a 1,2 y 17 horas después de ser liberados.

\section{Actividad insecticida de llama del bosque}

Obtención de extractos de hoja y flor de llama del bosque. Se pesó una muestra de $200 \mathrm{~g}$ de hojas secas molidas, estas fueron maceradas en aproximadamente $3 \mathrm{~L}$ de etanol al 95\% durante 7 días; las soluciones etanólicas obtenidas se reunieron, se filtraron y se concentraron a presión reducida en rotavapor, obteniendo $17.22 \mathrm{~g}$ de extracto de hojas, con un rendimiento de $8.61 \%$. Para el caso de las flores se pesaron para la extracción $74.11 \mathrm{~g}$ de material y se obtuvieron $11.77 \mathrm{~g}$ de extracto de flores, obteniendo un rendimiento de $15.88 \%$.

Procedimiento. En el ensayo se evaluaron tres concentraciones de extractos de hojas y flores de llama del bosque. Las concentraciones evaluadas fueron: 1.01 .5 y $2 \mathrm{mg}$ de extracto/ml de solución. Como disolvente se utilizó agua destilada y alcohol al $25 \%$. La solución se aplicó mediante un aspersor de polipropileno de $200 \mathrm{ml}$ de capacidad, se asperjaron $2 \mathrm{ml}$ por caja Petri. Se colocaron 10 gorgojos adultos de $S$. zeamais sobre papel filtro Watmann \# 1, en una placa Petri. Las condiciones experimentales fueron las mismas que en el ensayo de repelencia. En este ensayo se llevó registro del número de gorgojos muertos a 1, 2, 6 y 12 horas después de la aplicación de los extractos.

Diseño experimental. El diseño utilizado fue al azar, empleando 3 tratamientos y 4 repeticiones. A cada lectura se realizó un ANDEVA y una prueba de comparaciones múltiple de Tukey con un nivel de significancia del 5\% para establecer si existían diferencias estadísticamente significativas entre tratamientos. Los datos fueron transformados utilizando la fórmula de $(\mathrm{X}+1)^{1 / 2}$. El paquete estadístico utilizado fue el Minitab.

\section{RESULTADOS Y DISCUSIÓN}

Resultados de actividad repelente de gorgojos de maíz con hojas y flores de llama del bosque. La actividad repelente de las hojas y flores de llama del bosque presentó el mayor porcentaje a las 17 horas de la aplicación con el tratamientos de flores (79.37\%), estableciéndose diferencias significativas entre las flores y los demás tratamientos. A continuación se presentan los resultados del promedio porcentual acumulado de gorgojos que salieron de los vasos perforados en los diferentes tratamientos de hojas y flores frescas después de la aplicación (cuadro 1).

Cuadro 1. Promedio del porcentaje acumulado de insectos escapados de los vasos por actividad repelente de llama del bosque después de la aplicación

\begin{tabular}{llll}
\hline Tratamiento & $1 \mathrm{~h}$ & $2 \mathrm{~h}$ & $17 \mathrm{~h}$ \\
\hline Flores frescas & 56.87 & 68.75 & 79.37 \\
Hojas frescas & 53.12 & 58.12 & 63.75 \\
Testigo & 43.75 & 46.25 & 52.50 \\
\hline
\end{tabular}


En el cuadro 1, se muestra que el mejor tratamiento de efecto repelente de gorgojos del maíz fueron las flores frescas promedio del $79.37 \%$ de gorgojos escapados; el tratamiento de hojas frescas presentó el $63.75 \%$, ambos tratamientos son superiores al testigo el cual alcanzó un 52.50\% de gorgojos escapados a las 17 horas.

Cuadro 2. Valores de probabilidad final de los ANDEVA de la actividad repelente de llama del bosque

\begin{tabular}{lccc}
\hline Tiempo & $1 \mathrm{~h}$ & $2 \mathrm{~h}$ & $17 \mathrm{~h}$ \\
\hline Tratamientos & 0.132 & 0.011 & 0.005 \\
\hline
\end{tabular}

Los resultados de los ANDEVA determinaron que a una hora de toma de datos no hubo diferencia significativa) entre tratamientos ( $p=0.132$ ), (cuadro 2). Los porcentajes de gorgojos escapados también son similares (cuadro 1). A las dos horas se realizó un nuevo conteo acumulado de gorgojos escapados y en el ANDEVA se encontraron diferencias estadísticas significativas con un $5 \%$ de significancia $(\mathrm{P}=0.011)$. Por lo cual se realizó una prueba de comparaciones múltiple de Tukey con un nivel de significancia del 5\%, el mayor al efecto repelente fue el de las flores (cuadro 3). El promedio del porcentaje acumulado de gorgojos escapados también presentó la misma tendencia. La media más alta de gorgojos escapados por efecto repelente de llama del bosque fue para el tratamiento con flores, seguido de hojas y por último el testigo (cuadro 1).

A las 17 horas se efectuó la última toma de datos y los resultados acumulados del promedio de gorgojos escapados se sometieron a otro ANDEVA, el análisis arrojo diferencias significativas entre tratamientos con un $5 \%$ de significancia $(\mathrm{P}=0.005)$ por lo que se realizó una prueba de medias para determinar el tratamiento de mayor actividad repelente. En base a la prueba de medias, el mejor tratamiento con efecto repelente de gorgojos del maíz, a las 17 horas de liberados los gorgojos, fue las flores de llama del bosque, seguido de las hojas, resultados similares a los obtenidos a las dos horas de la aplicación.

Cuadro 3. Resultados la prueba de medias de Tukey del número de insectos escapados a las 17 horas de liberados en los tratamientos

\begin{tabular}{lccc}
\hline Tratamiento & Media & $\begin{array}{c}\text { Desviación } \\
\text { Estándar }\end{array}$ & Categoría \\
\hline Flores & 15.875 & 3.044 & $\mathrm{~A}$ \\
Hojas & 12.750 & 2.493 & $\mathrm{AB}$ \\
Testigo & 10.500 & 3.117 & $\mathrm{~B}$ \\
\hline
\end{tabular}

Resultados del efecto insecticida de extractos de hojas y flores de llama del bosque en gorgojos de maíz. En el cuadro 4, se presenta el promedio del porcentaje acumulado de mortalidad de gorgojos después de la aplicación de los extractos de hojas y flores de llama del bosque.
Cuadro 4. Promedio del porcentaje acumulado de mortalidad de gorgojos por actividad insecticida de los extractos de llama del bosque

\begin{tabular}{llrrrr}
\hline Tratamiento & & \multicolumn{1}{c}{$\mathrm{h}$} & \multicolumn{1}{c}{$\mathrm{h}$} & \multicolumn{1}{c}{$6 \mathrm{~h}$} & \multicolumn{1}{c}{$12 \mathrm{~h}$} \\
\hline Extracto de hojas & $1 \mathrm{mg} / \mathrm{ml}$ & 0.00 & 6.67 & 6.67 & 6.67 \\
Extracto de hojas & $1.5 \mathrm{mg} /$ & 0.00 & 6.67 & 6.67 & 10.00 \\
Extracto de hojas & $2.0 \mathrm{mg} / \mathrm{ml}$ & 0.00 & 0.00 & 0.00 & 0.00 \\
Extracto de flores & $1 \mathrm{mg} / \mathrm{ml}$ & 3.30 & 40.00 & 47.00 & 47.00 \\
Extracto de flores & $1.5 \mathrm{mg} / \mathrm{ml}$ & 3.30 & 70.00 & 70.00 & 70.00 \\
Extracto de flores & $2.0 \mathrm{mg} / \mathrm{ml}$ & 16.67 & 76.70 & 76.70 & 76.70 \\
Testigo & 0.00 & 8.00 & 8.00 & 8.00 & \\
\hline
\end{tabular}

Como puede observarse en el cuadro 4, el mayor número de insectos muertos se presentó en las unidades con aplicación de extractos de flores. La mayor mortalidad de gorgojos se presentó a las 12 horas de la aplicación, empleando las concentraciones de 1.5 y $2.0 \mathrm{mg} / \mathrm{ml}$, determinándose porcentajes de mortalidad de 70.0 y $76.7 \%$ respectivamente. Con los extractos de hojas no hubo efecto insecticida significativo bajo condiciones experimentales de laboratorio.

Los valores de probabilidad (P) de los ANDEVA arrojaron diferencias entre tratamientos con un 5\% de significancia (cuadro 5).

Cuadro 5. Valores de probabilidad final de los ANDEVA de la actividad insecticida de extractos de llama del bosque

\begin{tabular}{lcccc}
\hline Tiempo & $1 \mathrm{~h}$ & $2 \mathrm{~h}$ & $6 \mathrm{~h}$ & $12 \mathrm{~h}$ \\
\hline Tratamientos & 0.009 & 0.000 & 0.000 & 0.000 \\
\hline
\end{tabular}

A 1 hora de aplicados los extractos el ANDEVA determinó diferencias significativas entre tratamientos $(\mathrm{P}=0.009)$, también a las 2 horas $(\mathrm{P}=0.000), 6$ horas $(\mathrm{P}=0.000)$ y 12 horas de la aplicación de los extractos de llama del bosque $(\mathrm{P}==.000)$. El mejor resultado en cuanto al promedio del porcentaje acumulado de mortalidad de gorgojos se obtuvo con el extracto de flores a $2 \mathrm{mg} / \mathrm{ml}$ (cuadro 4). También, con base en la pruebas de prueba de comparaciones múltiple de Tukey con un nivel de significancia del $5 \%$, el mejor tratamiento con efecto insecticida de gorgojos del maíz a las 12 horas después de la aplicación de los extractos, fueron los extractos de flores de llama del bosque a $2 \mathrm{mg} / \mathrm{ml}$ seguido de la concentración de extracto de flores a $1.5 \mathrm{mg} / \mathrm{ml}$ (cuadro 6).

Cuadro 6. Resultados la prueba de medias de Tukey del número de insectos muertos a las 12 horas después de la aplicación de los tratamientos

\begin{tabular}{lccc}
\hline Tratamiento & Media & Desviación estándar & Categoría \\
\hline Flores $2.0 \mathrm{mg} / \mathrm{ml}$ & 2.9969 & 0.1669 & $\mathrm{~A}$ \\
Flores $1.5 \mathrm{mg} / \mathrm{ml}$ & 2.8134 & 0.3566 & $\mathrm{~A}$ \\
Flores $1.0 \mathrm{mg} / \mathrm{ml}$ & 2.2602 & 0.9149 & $\mathrm{AB}$ \\
Hojas $1.5 \mathrm{mg} / \mathrm{ml}$ & 1.3821 & 0.3671 & $\mathrm{BC}$ \\
Hojas $1.0 \mathrm{mg} / \mathrm{ml}$ & 1.2761 & 0.2391 & $\mathrm{BC}$ \\
Testigo & 1.2761 & 0.2391 & $\mathrm{BC}$ \\
Hojas $2.0 \mathrm{mg} / \mathrm{ml}$ & 1.0000 & 0.0000 & $\mathrm{C}$ \\
\hline
\end{tabular}


El mayor promedio acumulado del porcentaje de mortalidad de gorgojos con el extractos de flores a $2 \mathrm{mg} / \mathrm{ml}$ fue del $76.70 \%$ a las 12 horas de la aplicación (cuadro 4), bajo condiciones experimentales del laboratorio de Ecología Química de ECOSUR.

La pérdida de granos en almacenaje es uno de los problemas que enfrenta el agricultor en "post cosecha". Esto es especialmente importante en los países en desarrollo, donde los productores a pequeña escala ven mermadas sus cosechas a causa de la destrucción de los granos almacenados por roedores, insectos, hongos y bacterias (Larraín, 1994).

Por esta razón se hace necesario buscar alternativas para el pequeño agricultor que sean de bajo riesgo y de fácil acceso para el control de aquellas especies que atacan los granos almacenados. Una de estas opciones es el uso de plantas como la llama del bosque", la cual es una especie introducida y de amplia distribución.

De acuerdo a los resultados obtenidos del ANDEVA en el ensayo de repelencia a las 2 y 17 horas de la evaluación, se demostró que existieron diferencias estadísticas significativas entre los tratamientos ( $\mathrm{P}=0.011$ y 0.005$)$ de flores y hojas de llama del bosque en cuanto a la media acumulada del número de gorgojos escapados de los vasos con granos de maíz. Con base en la prueba de comparaciones múltiple de Tukey con un nivel de significancia del 5\%, el mejor efecto repelente para el gorgojo del maíz fue el de la aplicación de flores de llama del bosque; la repelencia a las 17 horas fue de $79.37 \%$ de gorgojos escapados; el tratamiento de hojas frescas reportó el $63.75 \%$, ambos tratamientos fueron superiores al testigo el cual alcanzó un $52.50 \%$.

En cuanto al efecto insecticida los extractos de flores a $2,1.5$ y $1.0 \mathrm{mg} / \mathrm{ml}$ de solución fueron los más efectivos presentando: $76.7 \%$, 70 y $47 \%$ de mortalidad respectivamente, también presentaron las medias más altas del número de gorgojos muertos. Los extractos de flores a 2.0 y $1.5 \mathrm{mg} / \mathrm{ml} \mathrm{de}$ solución presentaron la más alta mortalidad de gorgojos del maíz a las 2 y 12 horas después de la aplicación. Con base a los análisis de varianza existieron diferencias significativas entre los tratamientos $(\mathrm{P}=0.009,0.000,0.000$ y 0.000 a 1 , 2, 6 y 12 horas de la aplicación). La prueba de comparaciones múltiple de Tukey con un nivel de significancia del 5\%, determinó que los extractos de flores presentaron mayor actividad insecticida que los extractos de hojas de llama del bosque, en gorgojos de maíz (S. zeamais M.) a 2.0 y $1.5 \mathrm{mg} / \mathrm{ml}$.

Aunque los porcentajes de mortalidad presentados son menores a los obtenidos por Cubas (1995), que reporta el $92 \%$ de mortalidad con aceite de Eucalipto, seguido de la Soya con el $73 \%$, en una evaluación in vitro, en concentra- ciones de $2.0 \mathrm{ml}$ de aceite/ $\mathrm{kg}$ de semilla de maíz a las 48 horas de la aplicación. Los porcentajes de mortalidad de gorgojos con extractos de flores de llama del bosque son similares a los obtenidos con el aceite de Soya, por lo cual en función de la disponibilidad de material y costos los extractos de flores de llama del bosque, presentan una buena alternativa al control de gorgojos en maíz almacenado, especialmente en el área rural donde este árbol es abundante. Por lo que una opción para el control de plagas son las plantas con efecto insecticida que se encuentren en cada región, ya que son de fácil manejo y sus residuos son de vida corta (Lagunes, 1994).

Los extractos acuosos de llama del bosque se han evaluado sobre larvas de mosquitos Anopheles albimanus, con buenos resultados (Torres, Velazquez, Rios, Vasquez, Penilla y Rodriguez, 2010). También se ha demostrado que los extractos tienen efecto antibacteriano (Das \& Doss 2009) y antimalárico (Makinde, Amusan \& Adesogan (1998). Portugal-Araujo (1963) encontraron más de 200 insectos muertos en flores de $S$. campanulata, la mayoría eran abejas meliponas, moscas y hormigas. En cuanto a estudios del néctar de la planta, Trigo y Santos (2000), determinaron que en el mucílago contenido en los botones florales y en las flores jóvenes puede estar disuelto el néctar y ser el responsable de la muerte de insectos. En observaciones de campo se determinó que durante el día que las flores se abren (antesis), muere el $96.8 \%$ de insectos, en el segundo día la mortalidad disminuye $(2.3 \%)$ y del tercero al quinto día la mortalidad de insectos cae al 0.3\%. Segantini, Romano \& Ribeiro (2002), evaluaron el efecto de extractos acuosos de las hojas de $S$. campanulata al 1, 2, 3, 4 y 5\%, sobre larvas del segundo instar de Spodoptera frugiperda, realizaron lecturas de mortalidad de larvas a las 24, 36, 48 y 72 horas después de inicio del experimento en laboratorio y demostraron que a las 72 horas las concentraciones de 4 y $5 \%$ causaron una mortalidad del 91 al 100 \%. Davis, Pylatuik, Poradis, \& Low (1998), reportan la presencia de carbohidratos, proteínas, aminoácidos, iones inorgánicos y constituyentes aromáticos en el néctar, los cuales producen la toxicidad y pueden ser atrayentes para algunos organismos.

\section{AGRADECIMIENTOS}

Se agradece la colaboración del Laboratorio de Investigación de Productos Naturales de la Facultad de Ciencias Químicas y Farmacia de la USAC para la elaboración de extractos y del Laboratorio de Ecología Química de Insectos en ECOSUR, Tapachula, México por las facilidades brindadas para la realización de la actividad repelente e insecticida de llama del bosque contra gorgojos del maíz. 


\section{REFERENCIAS BIBLIOGRÁFICAS}

Davis, A; Pylatuik, J; Poradis, J; Low, N. 1998. Nectar carbohydrate and composition vary in relation to nectar anatomy and location whitin individual flowers of several species of Brassicaceae. Planta. 305-318.

Das, A; Dhanobalan, R; Doss, A. 2009. In Vitro antibacterial activity of two

medicinal plants against Bovine Udder Isolated Bacterial Pathogens from Dairy Herds. Ethnobotanical Leaflets. 13: 152-158.

Cubas, R. 1995. Control de Sitophilus oryzae L. (Coleóptera: Curculionidae) en maíz almacenado mediante el uso de aceites esenciales. In Valencia, L; De la Peña, E. Aportes al manejo ecológico de cultivos. Red de Acción Alternativa al uso de Agroquímicos (RAAA), Lima, Perú. p. 97-99.

García, J. 1997. Introducción a los plaguicidas. Ed. Universidad Estatal a distancia, Costa Rica. p 1-13.

Lagunes, TA. 1994. Extractos y polvos vegetales y minerales para el combate de plagas de maíz y frijol en la agricultura de subsistencia. Colegio de Posgraduados/USAID/CONACYT/BORUCONSA. Montecillo, Edo. De México 35 p.

Lagunes, T; Domínguez, R; Rodríguez, JC. 1985. Plagas del maíz en la meseta central de México. Colegio de Postgraduados. Universidad Autónoma Chapingo. Documento de trabajo. Montecillo. Texcoco. ME. 100 p.

Larraín, P. 1994. Manejo integrado de plagas en granos almacenados. Investigación y progreso agropecuario, La Platina v. 81. p. 10-16.

Levison, H. Levison, A. 1998. Control of stored food pests in the ancient Orient and classical antiquity, Journal of Applied Entomology 122:137-144.

Makinde, JM; Adesogan, EK; Amusan, OO. 1987. The Schizontocidal activity of Spathodea campanulata leaf extract on Plasmodium berghei berghei in mice. Phytoterapy Research. 1(2):65-68.

Makinde, JM; Amusan, OO; Adesogan, EK. 1998. Antimamalarial Active of Spathodea campanulata Steam Bark. Planta Medica. 10(8):692-693.

Matthews, GA. 1993. Insecticide application in stores. In: Mattews, G.A; Hislop, EC. Ed. Application Technology for Crop Protection. CAB International, Wallingford, UK. p. 305-315.

Mohan, S; Fields, P. 2002. A Simple techique to asses compounds that are repellent or attractive to stored-product insects. Journal of Stored Products Research 38:23-31.

Morgan, C; Sherington, J; Gudrups, I; Bowden, NS. 1998. The assessment of potential attractants to beetle pests: improvements to laboratory pitfall bioassay methods. Journal of Stored Prod. Res. 34:59-74.

Ngouela, S; Nyasse, B; Tsamo, E; Sondengam, BL; Connolly, JD. 1990. Spathodic acid: a triterpene acid from the stem bark of Spathodea campanulata. Plant Physiology and Biochemistry. 29(12):3959-3961.

Portugal-Araujo, V. 1963. O perigro de dispersao da Tuliperira do Gabao (Spathodea campanulata Beauv), Chacaras e Quintais. 107:562.

Saunders, J; Coto, D; King, A. 1998. Plagas invertebradas de cultivos anuales alimentícios en América Central. CATIE, CR. 127 p.

Segantini, BM; Romano, FC; Ribeiro, CC. 2002. Efeito insecticida de extracto aquoso de folhas de Spathodea campanulata (Bignonianceae) sobre Spodoptera frugiperda (J.E, Smith, 1797) Lepidoptera: Noctuidae em Laboratorio. Artigo do Facultade Cantareira, s.p. Brasil.

Silva G; Pizarro, D; Casals, P; Berti, M. 2003. Evaluación de plantas medicinales en polvo para el control de Sitophilus zeamais Motschulsky en maíz almacenado. Agrociencia, 9:384-385.

Silva-Aguayo GI; Coger-Melivilu, R; Hepp-Gallo, R; Tapia-Vargas, M. 2005. Búsqueda de plantas con propiedades insecticidas para el control de Sitophilus zeamais en maíz almacenado. Rev Agropec. BR. 40:11-17.

Subramanyam, Bh; Hagstrum, D. 1995. Resistence measurement and management. In: Subramanyam, Bh; Hagstrum, D. (Ed). Integrated Management of Insects in Stored Products. Marcel Dekker, Inc. New York, p. 331-398.

Tejeda, T. 1995. Control de gorgojo del maíz. En Valencia, L; De la Peña, E. Aportes al manejo ecológico de cultivos. Red de Acción Alternativa al uso de Agroquímicos (RAAA), Lima, PE. p 151-157.

Torres, E; Velásquez, G; Ríos, D; Vásquez, M; Penilla, N; Rodríguez, A. 2010. Toxicity of Spathodea campanulata P Beauvois (Scrophulariales: Bignoniaceae) aqueous extractas against immature stages of Anopheles albimanus (Diptera: Culicidae) under laboratory conditions. Research and Reports in Tropical Medicine. 1: 83-87.

Trigo, JR; Santos, WF. 2000 Insect Mortalitly in Spathodea campanulata Beauv. (Bignoniaceae) Flowers. Sao Carlos, Brasil. Rev. Bras. Biol. 60(3):537-538. 